\title{
Efficacy of Osteogenic Protein-1 in a Challenging Multilevel Fusion Model
}

\author{
Matthew J. Mermer, MD, ${ }^{*}$ Munish C. Gupta, MD, ${ }^{*}$ Donna L. Wheeler, PhD, $†$ \\ Joel Helgerson, PhD, † A. Hari Reddi, PhD, ${ }^{*}$ Scott Hazelwood, PhD, ${ }^{*}$ and \\ Daniel R. Benson, MD*
}

Study Design. A therapeutic study compared the influence of osteogenic protein-1 to autograft and collagen carrier in multilevel sheep spine fusions.

Objective. To evaluate the efficacy of osteogenic protein-1 compared to autograft and collagen carrier in achieving fusion in a challenging multilevel lumbar spine ovine model.

Summary of Background Data. Bone morphogenetic proteins can successfully augment spinal fusion. To date, all the preclinical and clinical studies using bone morphogenetic proteins have evaluated single-level fusion. In practice, multiple level fusions are commonly required for various conditions, like spinal deformity.

Methods. Eighteen sheep underwent three-level spine fusion. Six sheep were treated with osteogenic protein-1 and its carrier, autograft, or with the carrier alone. Specimens were analyzed for evidence of fusion by palpation, radiographic and histologic analysis, and biomechanical testing.

Results. Manual palpation testing for the presence of fusion showed none of the specimens fused all three levels or fused at the lumbosacral junction. No statistically significant difference was found between the osteogenic protein-1 and autograft groups' fusion rates based on radiographic grading $(P=0.65)$ or biomechanical testing. Histologic analysis showed no qualitative difference in bone morphology or cellularity of fusion masses when comparing the autograft and osteogenic protein-1 specimens.

Conclusions. No model before this exists that tests the efficacy of bone morphogenic proteins in as challenging an environment. Extrapolation of single-level preclinical and clinical studies with bone morphogenic proteins for use in multilevel fusion requires careful review. Autograft and osteogenic protein-1 had similar rates of fusion. A high rate of nonunion is seen with this multiple level fusion to the sacrum using autograft or osteogenic protein-1. The biologic enhancement with osteogenic protein-1 is not able to overcome this mechanically rigorous

From the "Department of Orthopaedic Surgery, University of California, Davis, Sacramento, Calfornia, and the †Department of Mechanical Engineering, Colorado State University, Fort Collins, Colorado. Support for this project was partially provided by Stryker Biotech (Hopkinton, MA).

Acknowledgment date: October 24, 2002. First revision date: March 18, 2003. Acceptance date: May 22, 2003.

The device(s)/drug(s) that is/are the subject of this manuscript is/are not FDA approved for this indication and is/are not commercially available in the United States.

Corporate/Industry funds were received to support this work. No benefits in any form have been or will be received from a commercial party related directly or indirectly to the subject of this manuscript.

Address correspondence and reprint requests to Matthew J. Mermer, MD, University of California, Davis, Department of Orthopaedic Surgery, 4860 Y Street, Suite 3800, Sacramento, CA 95817, USA; E-mail: mermerm@hss.edu model. [Key words: osteogenic protein-1, bone morphogenic proteins, multiple level spine fusion] Spine 2004;29: 249-256

Posterior spinal fusion is a commonly performed procedure for a variety of pathologies of the spine. Posterior approach is used for decompression of the neural elements and fusion with autograft and internal fixation. Nonunions and delayed unions still plague the method of posterior spinal fusion with overall rates as high as $40 \%$ and greater with multilevel fusions. ${ }^{1}$ Autograft has been used as the classic method for achieving posterolateral fusion. However, this method is associated with its own set of limitations. The amount of available autograft, its quality, pain at the donor site, infection, hemorrhage, nerve injury, and increased operative time contribute to the shortcomings of this standard. ${ }^{2-4}$ Much interest has been given to graft substitutes because of these problems. ${ }^{5}$ Osteoinductive proteins, such as recombinant human bone morphogenetic protein-2 and -7 (rhBMP-2 and rhBMP-7, respectively), have improved the results of single-level fusions in animal models. ${ }^{2,5-7}$ Bone morphogenic protein-7 (BMP-7), also known as osteogenic protein-1 (OP-1; Stryker Biotech, Hopkinton, MA), and BMP-2 have shown to decrease time to fusion, improve fusion rates, and have superior biologic and biomechanical outcomes compared with autogenous grafted fusions. $^{2,5-7}$ Data now exist from clinical studies showing BMPs to be more reliable than autograft for single level fusions. ${ }^{8}$

Multiple-level fusions are frequently performed for various spinal conditions, especially for spinal deformity correction. To date, none of the BMPs has been tested in multiple-level fusion models. The purpose of this work was to evaluate the efficacy of OP-1 compared to autograft in achieving fusion in a challenging multilevel lumbar spine ovine model.

\section{Materials and Methods}

Study Design. Eighteen sheep (Ovis aries) underwent bilateral posterolateral intertransverse process spine fusion of three levels from L5, L6, and L7 to S1 using a Wiltse-type approach (sheep spine have seven lumbar vertebrae). The facets and midline structures were not disturbed. Six sheep were treated with OP-1 (Stryker Biotech) and its carrier, six with autograft, and six with the carrier alone. Six months after unrestricted activity, the sheep were killed and the spines harvested en bloc from L4 to the sacrum. Six normal sheep spines were harvested from 
nonoperative control animals. The specimens were analyzed for evidence of fusion by radiographic grading, histology, and biomechanical and manual testing to compare the efficacy of OP-1 compared to autograft in achieving union. This protocol was approved by the University of California, Davis (U.C. Davis), Office of Environmental Health and Safety Animal Use and Care Committee.

Sheep Spine Fusion. Eighteen female nongravid mature western sheep were used for bilateral posterolateral lumbar spine fusion from L5 to the sacrum. The average sheep weight was $159.2 \mathrm{lbs}(72.4 \mathrm{~kg})$ with a range of $152-174 \mathrm{lbs}(69-79 \mathrm{~kg})$. Animals were sedated with intravenous pentothal (15-20 mg/ $\mathrm{kg}$ ), intubated, given $1 \mathrm{~g}$ of intramuscular cefazolin, placed prone in a well-padded position, and secured to a standard large animal operating table at the U.C. Davis Animal Research Facility.

The low back was sheared,and the skin was prepared with a povidone-iodine $10 \%$ scrub and draped. A single midline skin incision was made, followed by two parallel fascial incisions each about $2 \mathrm{~cm}$ lateral to midline from L5 to the sacrum. The transverse processes from L5 to L7 and the sacral ala were fully exposed, taking care not to disturb the facets or midline structures.

The bilateral posterior iliac crests were exposed through separate fascial incisions in six animals designated to the autograft group. For these sheep, an osteotome was used to remove the outer table of iliac crest and a curette was used to harvest $15 \mathrm{cc}$ of corticocancellous autograft from each side (total $30 \mathrm{cc}$ ). Bone wax was placed at the donor site. For all animals, a high-speed burr was used to decorticate the bilateral transverse processes from L5 to L7 and the sacral ala. The corticocancellous graft was placed in the gutter between the transverse processes of L5-L7 and from the transverse processes of L7 to the sacrum using $5 \mathrm{cc}$ per level (total $30 \mathrm{cc}$ ).

The six sheep assigned to the OP-1 group received the OP-1 and its carrier, which were combined during the surgery. Osteogenic protein-1 Putty (Stryker Biotech) contains $3.5 \mathrm{mg}$ OP-1 in $1 \mathrm{~g}$ bone-derived bovine type I collagen. Carboxymethylcellulose $(230 \mathrm{mg})$ was added to the putty by combining the contents of the putty additive with the OP-1 implant. The product was mixed with the addition of $2.5 \mathrm{cc}$ sterile saline for reconstitution. Each unilateral level received $3.5 \mathrm{mg}$ OP-1 in $1 \mathrm{~g}$ collagen/230 mg carboxymethylcellulose. For any given level, a total of $7.0 \mathrm{mg}$ OP-1 was used, and the resulting bilateral threelevel operative site contained $21.0 \mathrm{mg}$ of OP-1. Six sheep received the $1 \mathrm{~g}$ collagen/230 mg carboxymethylcellulose (without OP-1) per unilateral level.

The fascial and deep subcutaneous layers were closed using an absorbable braided running suture. The skin was approximated using staples. Povidone-iodine ointment and 5\% spray were used to cover the wound. Intramuscular methadone was given before extubating the animals. All sheep were allowed to heal for 6 months with unrestricted activity and then killed with pentobarbital.

Macroscopic Study. Spines were harvested from L4 to the sacrum. Soft tissues were sharply dissected away while taking care to preserve ligaments and leave the fusion mass undisturbed. Manual testing was performed before placing the specimens in $70 \%$ ethanol fixative. The appreciable distance between the tips of the spinous processes as viewed from the side was measured with a ruler during a nondestructive flexion- extension maneuver and recorded. If a change in the interspinous process distance greater than $2 \mathrm{~mm}$ was seen, then that particular level was recorded as a nonunion. Nonparametric data analysis using a Kruskal Wallis test followed by pair wise Mann Whitney tests was performed.

Each arthrodesis level was evaluated according to the method described by Guigui et al and assigned a palpation score. ${ }^{9}$ Accordingly, the fusion mass was palpated and recorded as continuous or discontinuous for each level. Those sites considered to have a continuous fusion mass were recorded as either thick (greater or equal to $2 \mathrm{~mm}$ in thickness) or thin fusions (less than $2 \mathrm{~mm}$ ). A thick and continuous fusion was assigned a value of 3 , a thin and continuous fusion received a value of 2 , and a discontinuous fusion site was assigned the value of 1 . Parametric analysis using a one-way analysis of variance (ANOVA) followed with a Bonferroni/Dunn post hoc test (multiple comparisons test) was performed. A nonparametric analysis was performed in the same manner as was described for the flexion-extension data as continuous fusions were compared to discontinuous ones.

Radiographic Analysis. Conventional radiographs were obtained of all sheep spines before surgery and after harvest ( 6 months postfusion). The Lenke grading system to evaluate in situ posterolateral fusions was used to grade each fusion level for the three groups. ${ }^{10}$ Letter grades were assigned to the fusion (grade $\mathrm{A}=$ definitely solid with bilateral stout fusion masses present; grade $\mathrm{B}=$ probably solid with a unilateral stout fusion mass and a contralateral thin fusion mass; grade $\mathrm{C}=$ probably not solid with a thin unilateral fusion mass and a probable pseudarthrosis on the contralateral side; and grade D = definitely not solid with thin fusion masses bilaterally with obvious pseudarthrosis or bone graft dissolution bilaterally). ${ }^{10}$ Three authors separately graded each fusion level blinded to the treatment. The majority grade was used for statistical analysis. The data were analyzed using a Kruskal Wallis nonparametric test followed by pair wise Mann Whitney tests. All statistical tests for the manual palpation and radiographic data were run at a significance level of $P=0.05$. Statistical power was obtained based on parametric data. The power for the nonparametric data were approximated based on the equivalent parametric power analysis.

Biomechanical Study. The 18 sheep spines in 70\% ethanol fixative were shipped via special courier to the Colorado State University for biomechanical testing. Six normal ovine spines served as biomechanical controls. The spines were potted in high strength dental plaster (Snapstone, WhipMix) in a base mold using a custom alignment jig. Three retroreflective markers were placed on each vertebral body using a centrally attached triad to monitor three-dimensional (3-D) rigid body motion of each vertebral body. All spines underwent 5 cycles of nondestructive loading with loads ranging from $-5.0 \mathrm{Nm}$ to 5.0 $\mathrm{Nm}$ in torsion and $0.0 \mathrm{Nm}$ to $5.0 \mathrm{Nm}$ in bending at a loading frequency of $1 \mathrm{~Hz}$. All spines were tested in all loading modes starting with torsion and followed by flexionextension, right lateral bending, and left lateral bending. Loading was applied to the spines at a constant loading rate using a biaxial servohydraulic material testing machine (MTS, Eden Prairie, MN). Pure torsion was applied along the longitudinal axis of the spine using a custom loading fixture. Pure bending was applied to the spine using a custom four-point bending 
fixture applying a constant bending moment across all three fusion sites. Relative interbody motion for each functional spinal unit (FSU) as well as overall motion (L5-sacrum) was quantified during loading using an optical kinematic system (Peak Performance Technologies, Englewood, CO). The last data cycle was processed for biomechanical analysis. The standard spine biomechanics parameters measured or calculated for each FSU included: stiffness ( $\mathrm{Nm} /$ degrees), neutral angle (degrees), and maximum angle (degrees). These parameters were measured or calculated for each loading orientation for each specimen. Stiffness was calculated as the linear region of the load-angular deformity curve for the bending tests. Stiffness in the torsion tests was calculated using the slope of the linear regression line of the load-angular deformity curve. The angle, during unloading, in which the moment or torque reached zero, was identified as the neutral angle for the bending and torsion tests, respectively. Maximum angle was the peak angular displacement during the loading cycle.

Biomechanical Data Analysis. The nonoperative specimens served as controls. Stiffness, neutral angle, and maximum angle values from experimental specimens (OP-1, autograft, and carrier) were normalized to the control specimens by calculating the difference from the experimental value from the mean control value. The normalized value was indicative of the difference from a nonoperative spine. Normalized values were summed for all loading conditions for each specimen to obtain a stiffness score. A cluster analysis was then used to determine the final overall "stiffness rank," which is based on the calculated stiffness, neutral angle, and maximum angle. A stiffness rank of 0 would indicate no fusion or high maximum angle and neutral angle with low stiffness. Similarly, a stiffness rank of 2 would indicate good fusion with minimal movement (maximum angle) and neutral angle and high stiffness. A stiffness rank of 1 would represent a moderate fusion with scores clustered between 0 and 2. A two-way ANOVA and multiple comparison procedures were used to determine differences in stiffness, neutral angle, and maximum angle for treatment groups and fusion level, whereas a Wilcoxon Sign-Rank test was used to determine differences in overall "stiffness rank." All statistical tests were run at a significance level of $P=0.05$.

Histologic Analysis. The four fused vertebral bodies were bisected in the sagittal plane using an Exakt Bone Saw (Exakt Technologies, Oklahoma City, OK). The right and left segments of fused spines were transversely sectioned at the L6 transverse process. Care was taken to cut down the center of the L6 transverse process so that the fusion mass between L6 and L5 and the fusion mass between L6 and L7 were preserved. This sectioning scheme provided the three-level fusion to be subdivided into a cranial fusion segment (L5-L6) and a caudal fusion segment (L6-L7-S1). Spines were fixed in 70\% ethyl alcohol (EtOH) immediately after harvesting. After fixation in fresh $70 \% \mathrm{EtOH}$ for 1 week, the specimens were dehydrated in graded solutions of $\mathrm{EtOH}(70 \%, 95 \%$, and $100 \%)$ and xylene over the course of 3 weeks. The samples were then infiltrated with a series of solutions containing methylmethacrylate, dibutylphthalate, and benzoyl peroxide. The final methylmethacrylate solution was polymerized to create hardened plastic blocks. From each specimen segment, two histologic blocks were generated, medial and lateral, and one $300 \mu \mathrm{m}$ histologic section was taken from each block using an Exakt diamond blade bone saw. All sections were ground using an Exact mi- crogrinder to 10 to $20 \mu \mathrm{m}$ thickness and stained using a modified Van Gieson bone stain with a Sanderson counterstain. Both low magnification and high magnification images were taken to illustrate fusion success and osseous morphology of the fusion mass. High-resolution digital images were acquired using an Image Pro Imaging system (Media Cybernetics, Silver Spring, MD), a Nikon E800 microscope (AG Heinze, Lake Forest, CA), Spot digital camera (Diagnostic Instruments, Sterling Heights, MI), and a Pentium IBM-based computer with expanded memory capabilities (Dell Computer Corp., Round Rock, TX). Qualitative histologic evaluations were made of all specimens to assess fusion site morphology, cellularity, and extent.

\section{- Results}

\section{Macroscopic Results}

All animals survived the surgery with no neurologic injuries or infections.

No fusions were observed at any level in the group treated with carrier alone. Gross motion was produced between adjacent spinous processes during flexionextension testing in all Carrier specimens.

In the autograft group, 23 fusion sites out of 36 received a palpation score of 1 (noncontinuous), 11 out of 36 received a score of 2 (thin and continuous), and 2 out of 36 received a 3 (thick and continuous). In the OP-1 group, 21 fusion sites out of 36 received a palpation score of 1,8 out of 36 received a score of 2 , and 7 out of 36 received a score of 3 (Figure 1 ). There was no difference between right and left sides within any group $(P>$ 0.05 , power $=0.96$ ) .

The autograft and OP-1 groups showed significantly greater palpation scores compared to the carrier group based on parametric analysis $(P=0.01$ for autograft $v s$. carrier; $P=0.0005$ for OP-1 vs. carrier, power $=0.93$ ). Nonparametric analysis also showed that the autograft group and OP-1 group had significantly greater palpation scores compared to the carrier group $(P=0.03$, power approximately 0.8 ). There was no significant difference between the autograft and OP-1 groups based on parametric and nonparametric analysis of the palpation scores $(P=$ 0.24 and $P=0.63$, respectively). The difference between fusion levels was not quite statistically significant within the autograft or OP-1 groups based on parametric and nonparametric testing $(P=0.055$, and $P=0.08$, respectively) for the palpation scores (Figure 1).

No motion or micromotion $(<2 \mathrm{~mm})$ during flexionextension testing was observed between the spinous processes in 8 out of 18 levels for the autograft group and 10 out of 18 levels in the OP-1 group. The autograft and OP-1 groups had significantly less motion when compared to the carrier group for the flexion and extension test $(P=0.01)$. There was no significant difference between the autograft and OP-1 groups $(P=0.57)$.

Analysis of the flexion and extension test between levels within each group was performed. In the autograft group, the L6-L7 level showed less motion during the flexion-extension test when compared to the L7-S1 level $(P=0.02)$. The OP-1 group was the only group to show micromotion at the lumbosacral junction in two cases, 


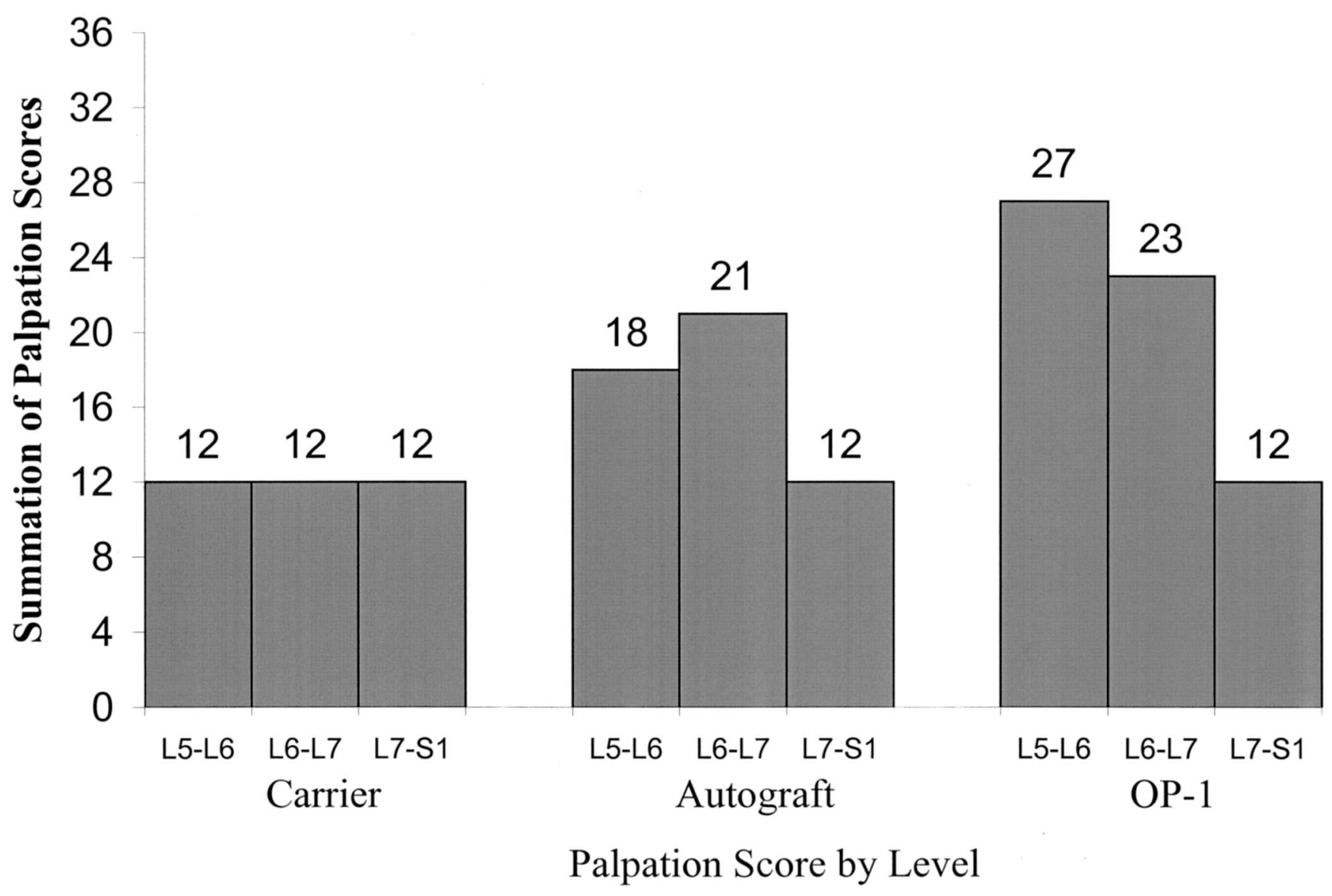

Figure 1. Summation of palpation scores for each level in the carrier, autograft, and OP-1 groups. A score of 1, 2, or 3 was given for each unilateral fusion site (see text). The lowest possible summation of scores $=12$; the greatest $=36$.

whereas gross motion was observed in all other specimens. No other significant differences were seen in the flexion-extension test between levels within a given group $(P>0.05)$.

\section{Radiographic Results}

Radiographic analysis of each level using the Lenke grading system showed the autograft group with 2 (A), 5 (B), $7(\mathrm{C})$, and 4 (D) fusions (7 out of 18 levels were either
Figure 2. Conventional radiographs of autograft group 6 months postfusion. Numbers in parentheses correspond to experimental number during study.

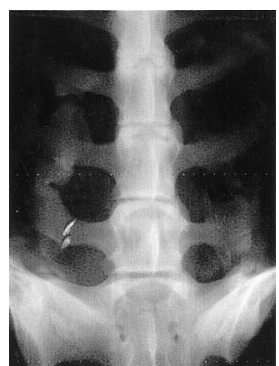

Autograft \#1 (\#39)

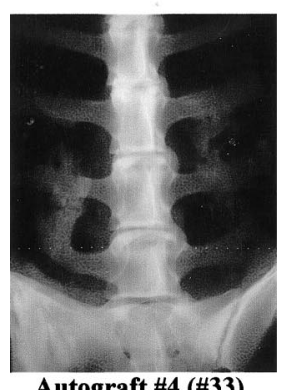

Autograft \#4 (\#33)

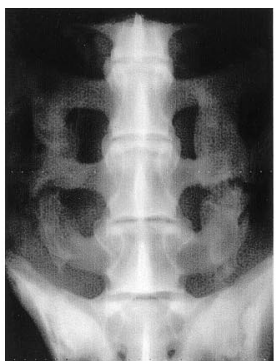

Autograft \#2 (\#31)

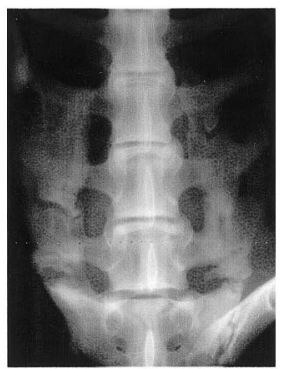

Autograft \#5 (\#37)

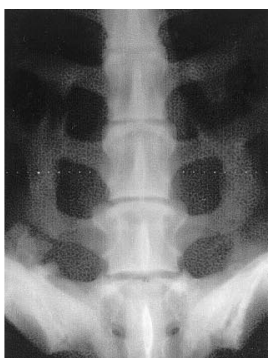

Autograft \#3 (\#32)

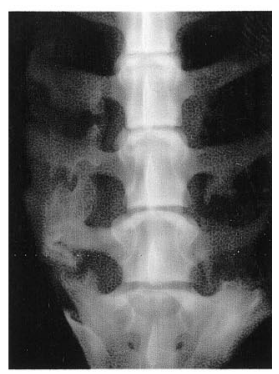

Autograft \#6 (\#42) 


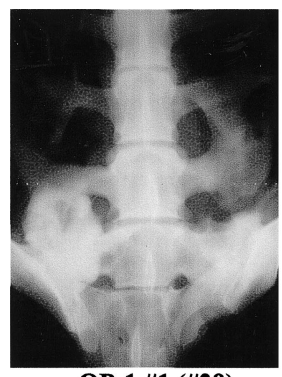

OP-1 \#1 (\#29)

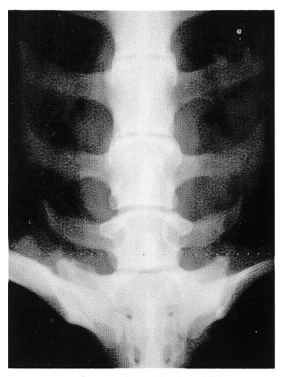

OP-1 \#4 (\#49)

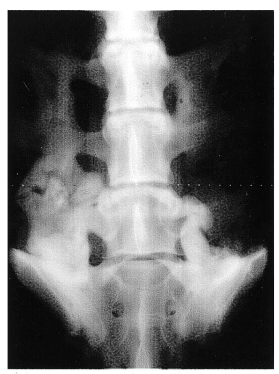

OP-1 \#2 (\#45)

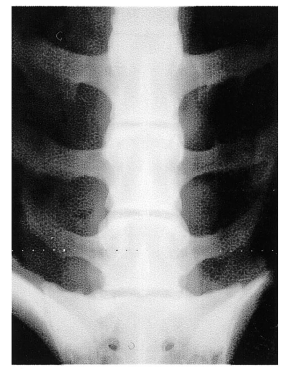

OP-1 \#5 (\#40)

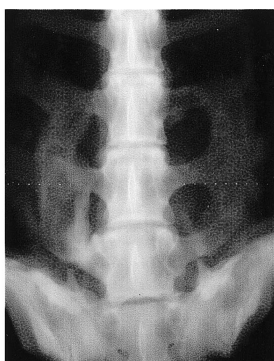

OP-1 \#3 (\#47)

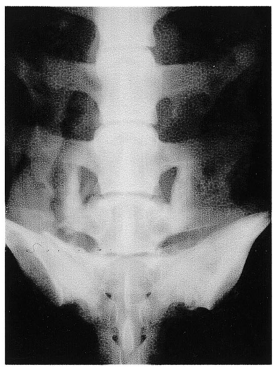

OP-1 \#6 (\#38)
Figure 3. Conventional radiographs of OP-1 Group 6 months postfusion. Numbers in parentheses correspond to experimental number during study. fused or probably fused; A's and B's) (Figure 2). The OP-1 group had 4 (A), $4(\mathrm{~B}), 1(\mathrm{C})$, and 9 (D) fusions (8 out of 18 fused or probably fused segments) (Figure 3). The carrier group had 18 grade D fusions (all levels) (Figure 4). There was a statistically significant difference between both the autograft compared to carrier and OP-1 compared to carrier radiographic grades $(P=$ $0.0005)$. There was no significant difference between the autograft and OP-1 groups' radiographic grades $(P=$ $0.65)$.

Analysis was performed to determine if there was a differences in grades between levels within each group. The autograft group had significantly higher fusion grades at the L6-L7 level when compared to the lumbo- sacral junction at L7-S1 $(P=0.008)$. No significant difference was observed between other levels within the autograft, OP-1, or carrier groups $(P>0.05)$.

\section{Biomechanical Results}

Biomechanical testing confirmed observations of gross motion between all functional spinal units (FSUs) in the nonoperated specimens and those that received carrier alone. Table 1 shows the number of spines and total percent of all FSUs with each stiffness rank by treatment and level for the autograft and OP-1 groups. Specimens treated with autograft showed 3 FSUs with overall stiffness ranks of 2 , very stiff $(17 \%), 7$ with ranks of 1 , moderately stiff (39\%), and 8 with ranks of 0 , not stiff
Figure 4. Number of levels receiving a particular Lenke radiographic grade in carrier, autograft, and OP-1 groups.

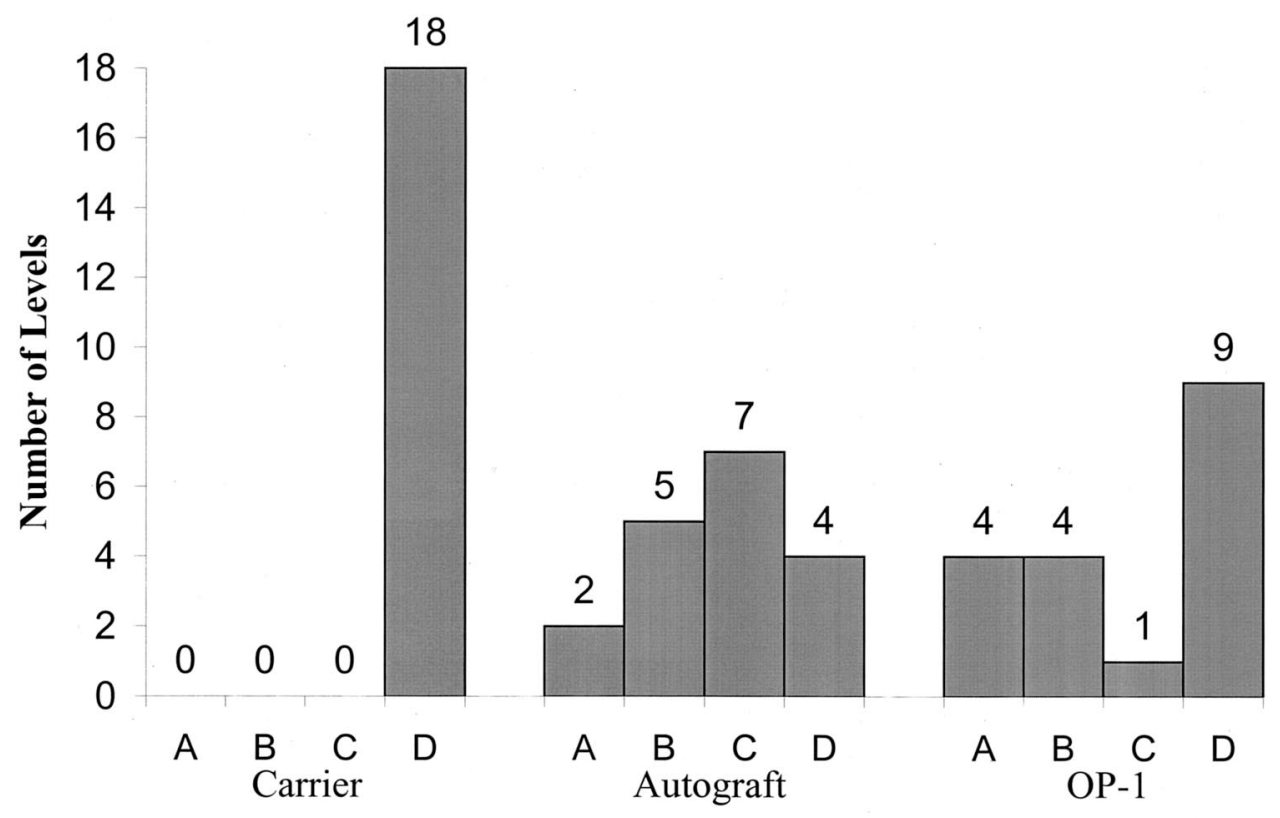

Radiographic Grade 
Table 1. Biomechanical Stiffness Rank

\begin{tabular}{lcccccccc}
\hline Group & ID & L5-L6 & L6-L7 & L7-S1 & L5-S1 & Rank $=2$ & Rank $=1$ & Rank $=0$ \\
\hline Autograft & 31 & 0 & 0 & 0 & 0 & $17 \%$ & $39 \%$ & $44 \%$ \\
& 32 & 1 & 1 & 1 & 2 & & & \\
& 33 & 0 & 0 & 0 & 0 & & & \\
& 37 & 2 & 2 & 1 & 2 & & & \\
& 39 & 2 & 1 & 0 & 2 & & & \\
0 OP-1 & 42 & 1 & 1 & 0 & 1 & & & \\
& 29 & 1 & 1 & 2 & 2 & $33 \%$ & $22 \%$ & $44 \%$ \\
& 38 & 0 & 2 & 0 & 1 & & & \\
& 40 & 0 & 0 & 0 & 0 & & & \\
& 45 & 2 & 2 & 2 & 2 & & & \\
& 47 & 1 & 2 & 1 & 2 & & &
\end{tabular}

Stiffness rank by level and for all three levels. ID corresponds to experimental number during study.

OP-1 = osteogenic protein-1.

(44\%). The spines treated with OP-1 showed 6 FSUs with overall stiffness ranks of $2(33 \%)$, 4 with ranks of 1 $(22 \%)$, and 8 with ranks of $0(44 \%)$ (Figure 5$)$. Those spines fused with either OP-1 or autograft had significantly greater overall stiffness and lower overall maximum angle and overall neutral angle when compared to nonoperative control or carrier group $(P<0.05)$. Stiffness ranks were significantly greater for the OP-1 and autograft groups compared to the nonoperative and carrier groups $(P<0.05)$. Although the OP-1 group had the greatest number of FSUs with a stiffness rank of 2, this was not statistically significant. There was no significant difference in any of the biomechanical parameters between the autograft and OP-1 groups $(P>0.05)$.

\section{Histologic Observations}

No qualitative difference was noted in bone morphology or cellularity of fusion masses when comparing the autograft and OP-1 specimens (Figure 6). High power pho-
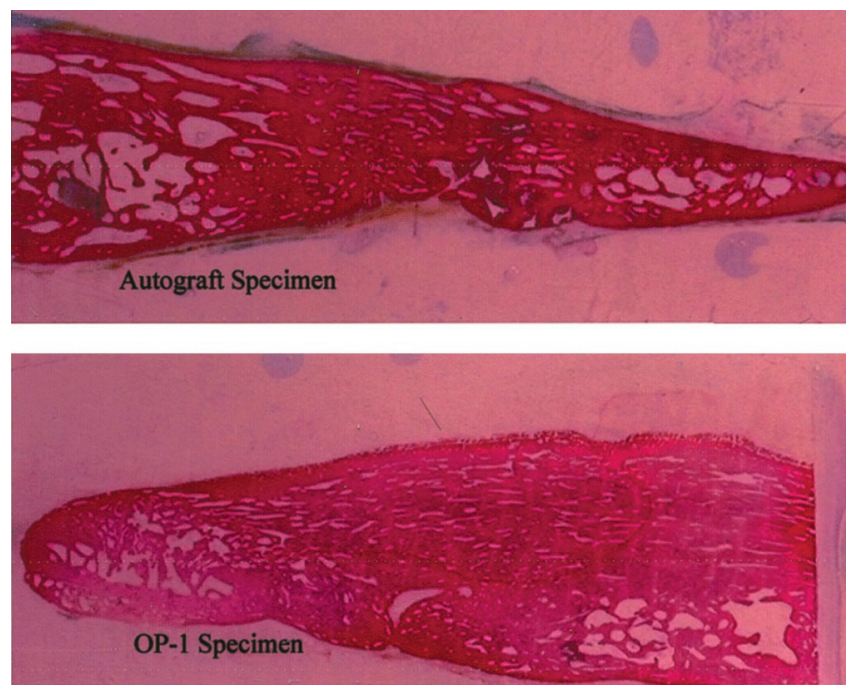

Figure 6. Low power photomicrographs of autograft and OP-1 fusion levels. These particular sections were each obtained from the L5-L6 level in both the autograft and OP-1 specimens.

tomicrographs showed successful fusion levels to consist of normal trabecular bone formation with osteoblasts and osteoid in both autograft and OP-1 specimens (Figure 7). Nonunion sites in both groups failed to demonstrate sufficient new bone formation and consisted largely of cartilage islands surrounded by fibroblasts.

\section{- Discussion}

Posterior spinal fusion is frequently performed for various types of spinal pathology, including spinal deformity. Nonunions or pseudarthroses in multiple levels still occur even when autograft and internal fixation are used. ${ }^{11,12}$ The incidence of pseudarthrosis for posterolateral lumbar intertransverse fusions ranges in the litera-
Figure 5. Number of levels receiving a particular stiffness rank in carrier, autograft, and OP-1 groups.

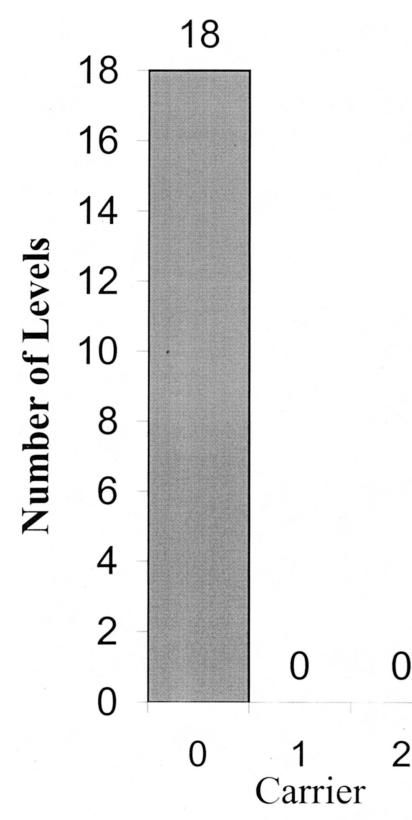

8

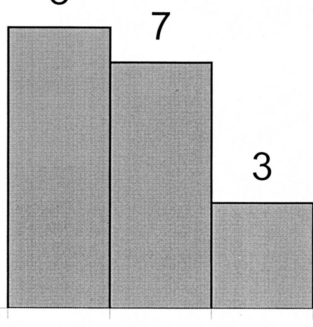

$0 \underset{\text { Autograft }}{1}$

Stiffness Rank 


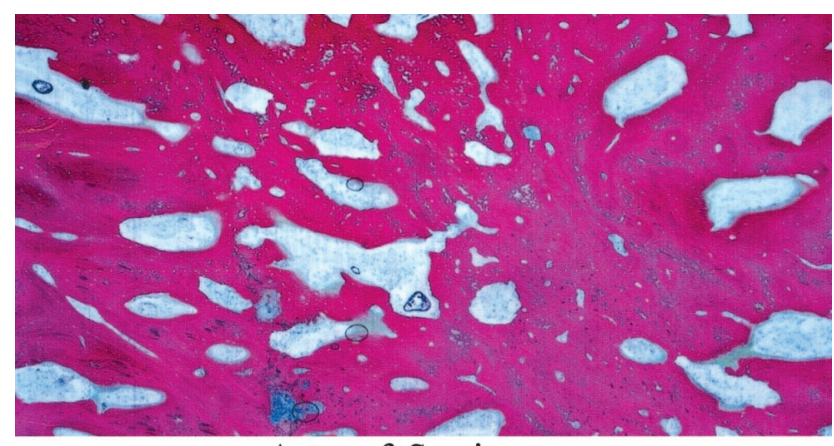

Autograft Specimen

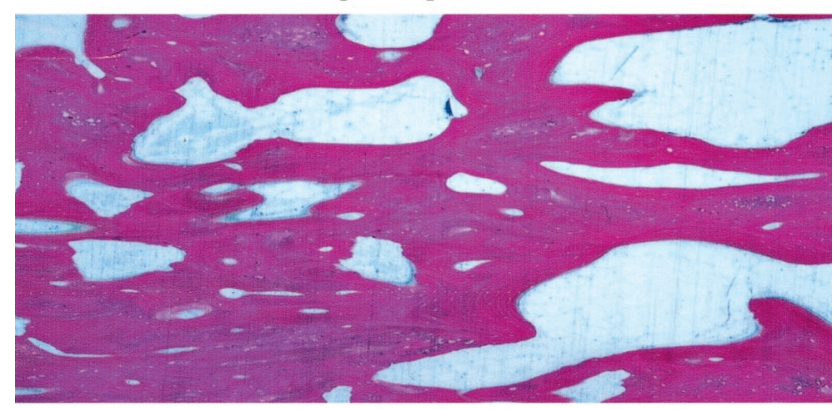

OP-1 Specimen

Figure 7. High power photomicrographs of fusion sites from autograft and OP-1 specimens. Both sections were obtained from the L5-L6 level.

ture from $3 \%$ to $35 \% .^{3,13,14}$ Deguchi et al were able to show an $82 \%$ fusion rate for single-level lumbar decompressions and posterolateral fusions in a retrospective clinical study where instrumentation was used in nearly every case. ${ }^{11}$ The rate fell to $74 \%$ for two-level fusions. ${ }^{11}$

A successful spinal fusion requires a mechanically stable environment as well as a biologically ideal fusion bed. A host of systemic factors, such as smoking, hormones, and nutrition, also influences this process. ${ }^{14}$ The use of BMPs as bone graft substitute has gained considerable interest in the recent past. Much work has evaluated the use of BMPs and their efficacy in augmenting spinal fusion. ${ }^{2,5-8,12-19}$ It has been demonstrated that BMPs may initiate all of the molecular mechanisms required for bone induction. ${ }^{15,16}$ The induction is then dependent on a necessary complement of extracellular matrix scaffolding. ${ }^{15,16}$ These two necessary components, growth factor plus matrix, along with an undifferentiated stem cell population, makeup the foundation for a potential fusion bed. ${ }^{14-16}$ The next critical ingredient for successful fusion would appear to be mechanical stability. As in the case of long bone fracture healing in a young person with a favorable biologic environment, the difference between union and nonunion may only be a stable versus unstable fracture pattern. Instrumentation has been shown to increase fusion rates in the lumbar spine. ${ }^{12,20}$ Our sheep model investigated whether BMPs (OP-1) could overcome the mechanically challenging environment of a multilevel fusion to the lumbosacral junction.

Care should be taken when extrapolating single-level fusion data with BMPs to multiple levels. Multiple-level lumbar fusion to the sacrum has a mechanically interactive fusion process between the levels. The timing of one level's success may contribute an adjacent level's failure.

Cook et al used a dog model to suggest the efficacy of rhOP-1 as a bone graft substitute for achieving stable lumbar single-level unilateral facet fusions. ${ }^{6}$ Their work also implied that rhOP-1 provided significantly more rapid fusion than did autogenous bone. ${ }^{6,17}$ Boden and Schimandle, Boden and Sumner, and Muschler et al showed that rhBMP-2 was as effective as autograft in a dog posterior lumbar spinal model. ${ }^{13,14,18}$ The results from both of these models have been variably interpreted because multiple interventions took place within the same animal. ${ }^{13-16}$

The rabbit model has been used by Boden et al and Schimandle et al to show the efficacy of bone protein extract and rhBMP-2 in lumbar intertransverse process spine fusions, respectively. ${ }^{16,19}$ The work of Schimandle et al demonstrated accelerated bone formation, consolidation, and remodeling compared to autogenous bone graft alone. ${ }^{19}$ It also showed that high dose rhBMP-2 and type I bovine collagen resulted in arthrodeses that were biomechanically superior to the other fusion techniques. ${ }^{19}$ The methodology developed in the Boden et al rabbit posterolateral lumbar model was transferred successfully to a nonhuman primate model. ${ }^{16}$ All of these models were for single-level fusions.

This multilevel lumbar sheep model demonstrated that neither autograft nor OP-1 could achieve fusion at all three levels. No significant difference was seen in manual testing, biomechanical testing, or radiographic grading between the autograft group and OP-1 group. Neither the autograft group nor the OP-1 group was able to demonstrate solid fusion at the lumbosacral junction (L7-S1). Osteogenic protein-1-treated fusions tended to be stiffer and more robust as observed in manual and biomechanical testing. The OP-1 group was also the only group to approach a solid fusion at the lumbosacral junction (L7-S1) out of all the specimens. To our knowledge, no work exists that evaluates the relationship between adjacent fusion levels. Other than the radiologic grades for the L6-L7 level compared to the lumbosacral junction in the autograft group, no statistically significant difference was seen between the fusion sites within any particular group. However, a trend did exist in both the autograft and OP-1 groups when comparing the proximal two fusion levels to the lumbosacral junction (Figure 1). This appeared to be a mechanical phenomenon.

\section{- Conclusion}

No model before this exists that tests the efficacy of OP-1 in as challenging an environment using multiple levels. Neither autograft nor OP-1 could fuse all three levels in any specimen. Autograft and OP-1 had similar rates of fusion. Although the OP-1-treated fusions tended to be stiffer and more robust, this could not be shown as a statistically significant difference. The biologic enhancement with OP-1 was not able to overcome this mechan- 
ically rigorous model. Extrapolation of single-level preclinical and clinical studies with BMPs for use in multilevel fusion requires further investigation.

\section{- Key Points}

- All the preclinical and clinical studies using BMPs have evaluated single-level fusion.

- No statistically significant difference was found between the OP-1 and autograft groups' fusion rates based on manual palpation, radiographic grading, biomechanical testing, or histologic analysis.

- A high rate of nonunion is seen with this multiple level fusion to the sacrum using autograft or OP-1.

- Extrapolation of single-level preclinical and clinical studies with BMPs for use in multilevel fusion requires careful review.

\section{Acknowledgments}

The authors thank Paul Cammack, MD, Ivan Cheng, $\mathrm{MD}$, and Russell Nelson, MD, for their contributions to this manuscript.

\section{References}

1. Cotler JM, Star AM. Complications of spinal fusions. In: Cotler JM, Cotler HB, eds. Spinal Fusion: Science and Technique. New York, NY: SpringerVerlag; 1990:361-87.

2. Sandhu HS, Kanim LEA, Kabo JM, et al. Evaluation of rhBMP-2 with an OPLA carrier in a canine posterolateral (transverse process) spinal fusion model. Spine 1995;20:2669-82.

3. Steinmann JC, Herkowitz N. Pseudoarthrosis of the spine. Clin Orthop 1992;284:80-90.
4. Summers BN, Eisenstein SM. Donor site pain from the ilium. A complication of lumbar spine fusion. J Bone Joint Surg Br 1989;71:677-80.

5. Cook SD, Rueger DC. Osteogenic protein-1: biology and applications. Clin Orthop 1996;324:29-38.

6. Cook SD, Dalton JE, Tan EH, et al. In vivo evaluation of recombinant human osteogenic protein (rhOP-1) implants as a bone graft substitute for spinal fusions. Spine 1994;19:1655-63.

7. Minamide A, Tamaki T, Kawakami M, et al. Experimental spinal fusion using sintered bovine bone coated with type I collagen and recombinant human bone morphogenetic protein-2. Spine 1999;24:1863-72.

8. Boden S, Zdeblick T, Sandhu H, et al. Spine 2000;25:376-381.

9. Guigui P, Plais PY, Flautre B, et al. Experimental model of posterolateral spinal arthrodesis in sheep. Spine 1994;19:2791-7.

10. Lenke L, Bridwell KH. Adult spondylolisthesis with lysis. In: Bridwell KH, DeWald R, eds. The Textbook of Spinal Surgery. Philadelphia, PA: Lippincott-Raven; 1997:1269-98.

11. Deguchi M, Rapoff AJ, Zdeblick T. Posterolateral fusion for isthmic spondylolisthesis in adults: analysis of fusion rates and clinical results. J Spinal Disord 1998;11:459-64.

12. Zdeblick T. A prospective, randomized study of lumbar fusion. Preliminary results. Spine 1993;18:983-91.

13. Boden S, Schimandle J. Biologic enhancement of spinal fusion. Spine 1995; 20:113S-23S.

14. Boden S, Sumner D. Biologic factors affecting spinal fusion and bone regeneration. Spine 1995;20:102S-112S.

15. Boden S, Schimandle M, Hutton W, et al. The use of an osteoinductive growth factor for lumbar spinal fusion. Part I: Biology of spine fusion. Spine 1995;20:2626-32.

16. Boden S, Schimandle M, Hutton W. The use of an osteoinductiove growth factor for lumbar spinal fusion. Part II: Study of dose, carrier, and species. Spine 1995;20:2633-44.

17. Cunningham BW, Kanayama M, Parker L, et al. Osteogenic protein versus autologous interbody arthrodesis in the sheep thoracic spine. Spine 1999;24: 509-18.

18. Muschler G, Hyodo A, Manning T, et al. Evaluation of human bone morphogenetic protein 2 in a canine spinal fusion model. Clin Orthop 1994;308: 229-40.

19. Schimandle JH, Boden SD, Hutton WC. Experimental spinal fusion with recombinant human bone morphogenetic protein-2 (rhBMP-2). Spine 1995; 20:1326-37.

20. Kotani Y, Cunningham BW, Cappuccino A, et al. The role of spinal instrumentation in augmenting lumbar posterolateral fusion. Spine 1996;21:278-87. 